\title{
FERROFLUID SQUEEZE FILM FOR SPHERICAL AND CONICAL BEARINGS
}

\author{
DINESH KUMAR, PRAWAL SINHA and PEEYUSH CHANDRA \\ Department of Mathematics, Indian Institute of Technology, Kanpur-208 016, India
}

\begin{abstract}
This paper analyzes ferrofluid squeeze film for spherical and conical bearings with a constant external magnetic field applied in the direction transverse to that of fluid flow. However, in this analysis, which accounts for the rotation of magnetic particles, no restriction is imposcd on magnetization vector. The governing equations of motion are nonlinear coupled equations and are solved using perturbation method in terms of non-dimensional Brownian relaxation time parameter. The effect of magnetic fluid parameters on various bearing characteristics is studied numerically.
\end{abstract}

\section{INTRODUCTION}

Ferrofluids are stable colloidal suspensions of very fine magnetic particles in a viscous fluid (carrier fluid). Such fluids can be positioned, confined or controlled at desired places under externally applied magnetic field. The external magnetic field also gives rise to an increase in effective viscosity of ferro solutions [1]. This has led to increasing application of ferrofluids as lubricants in bearings. In recent years, several authors have theoretically investigated characteristics of various bearing configurations using ferrofluid lubricants [2-9]. In particular, Verma [5] studied squeeze film between two rectangular parallel plates with lower plate bcing porous. It may be pointed out here that most of the above mentioned studies are based on the model of Neuringer and Rosensweig [10] which assumes that the magnetization vector is parallel to the applied magnetic field. This restriction is relaxed in the model of Shliomis [11] which takes into account the particle rotation also. Thus, Shukla and Kumar [8] studied characteristics of infinitely long squeeze film and slider bearing; while, Kumar and Chandra [9] analysed squeeze film with non-Newtonian magnetic fluids, using Shliomis model when external field is transversely applied. However these analyses are restricted for small magnetization vector such that the product terms of magnetization vector could be neglected.

In the present paper, we have studied ferrofluid squeeze film for spherical and conical bearings using perturbation analysis. The rotation of magnetic particles has been accounted for, by using the governing equations given by Shliomis [11], and no restriction is placed on magnetization vector. However, the applied magnetic field is assumed to be constant and imposed in the direction transverse to the fluid flow.

\section{GOVERNING EQUATIONS}

In recent years various theoretical models have been put forward to study the continuum description of ferrofluid flow. However, most of the analytical studies concerning the motion of ferrofluids are based on the formulation given by either Neuringer and Rosensweig [10] or Shliomis [11]. Neuringer and Rosensweig [10] developed a fairly simple model where the effect of magnetic body force was considered under the assumption of magnetization vector $\mathbf{M}$ being parallel to the magnetic field vector $\mathbf{H}$. Shliomis [11] in his formulation removed this assumption. Further, he pointed out that the magnetic particles in the fluid have Brownian motion and their rotation affects the motion of ferrofluids. Thus, Shliomis derived the equation of motion for ferrofluids by considering internal angular momentum $\mathbf{S}$ due to the self-rotation of particles. These equations governing the flow are given as follows [11]: 
Equation of motion:

$$
\rho \frac{\mathrm{d} \mathbf{U}}{\mathrm{D} t}=-\nabla P+\eta \nabla^{2} \mathbf{U}+\mu_{0}(\mathbf{M} \cdot \boldsymbol{\nabla}) \mathbf{H}+\frac{1}{2 \tau_{s}} \nabla \times(\mathbf{S}-I \mathbf{\Omega})
$$

Equation for internal angular momentum:

$$
\frac{\mathrm{DS}}{\mathrm{D} t}=\mu_{0} \mathbf{M} \times \mathbf{H}-\frac{1}{\tau_{S}}(\mathbf{S}-I \mathbf{\Omega})
$$

Equation for magnetization:

$$
\frac{\mathrm{D} \mathbf{M}}{\mathrm{D} t}=\frac{1}{I}(\mathbf{S} \times \mathbf{M})-\frac{1}{\tau_{B}}\left(\mathbf{M}-M_{0} \frac{\mathbf{H}}{|\mathbf{H}|}\right)
$$

Equation of conservation:

$$
\boldsymbol{\nabla} \cdot \mathbf{U}=0
$$

where $I$ is sum of moments of inertia of the particles per unit volume, $\tau_{s}$ is Relaxation time parameter, $\tau_{B}$ is Brownian relaxation time, and $\mu_{0}$ is permeability of free space.

For steady viscous flow of a ferrofluid equations (1)-(3) on ignoring inertial and angular dispersion terms reduce to [12]

$$
\begin{gathered}
-\nabla P+\eta \nabla^{2} \mathbf{U}+\mu_{0}(\mathbf{M} \cdot \nabla) \mathbf{H}+\frac{1}{2 \tau_{S}} \nabla \times(\mathbf{S}-I \mathbf{\Omega})=0 \\
\mathbf{S}=I \mathbf{\Omega}+\mu_{0} \tau_{S}(\mathbf{M} \times \mathbf{H}) \\
\mathbf{M}=M_{0} \frac{\mathbf{H}}{|\mathbf{H}|}+\frac{\tau_{B}}{I}(\mathbf{S} \times \mathbf{M})
\end{gathered}
$$

Substituting equation (6) in equation (5) gives

$$
-\nabla P+\eta \nabla^{2} \mathbf{U}+\mu_{0}(\mathbf{M} \cdot \boldsymbol{\nabla}) \mathbf{H}+\frac{\mu_{0}}{2} \nabla \times(\mathbf{M} \times \mathbf{H})=0
$$

While using equations (6) and (7), we get

$$
\mathbf{M}=M_{0} \frac{\mathbf{H}}{|\mathbf{H}|}+\tau_{B} \mathbf{\Omega} \times \mathbf{M}+\frac{\mu_{0} \tau_{B} \tau_{S}}{I}(\mathbf{M} \times \mathbf{H}) \times \mathbf{M}
$$

\section{SPHERICAL BEARING}

In this section we consider steady viscous flow of an incompressible ferrofluid between two spherical surfaces in relative normal motion (Fig. 1). A constant magnetic field is applied in a direction transverse to the motion of the fluid. The clearance $C$ (the difference between the radii, i.e. $R_{2}-R_{1}$ ) between the two spherical surfaces is small compared to the radius of the inner sphere. The inner sphere moves downwards with a normal velocity $V_{0}$ towards the external stationary sphere. The velocity $V_{0}$, which in general, is imparted by the load $W$, is considered constant in magnitude and direction, but is a function of time. The bearing is assumed infinitely long in axial direction and the film thickness is small compared to the radius, so the curvature of the fiuid film is neglected. Therefore the necessity of using cylindrical coordinates is eliminated and the Cartesian coordinate system can be used with $X=R_{1} \theta$.

Further, to simplify the analysis, we make following assumptions as considered by Walker and Buckmaster [3] and Shukla and Kumar [8]:

(i) The plates are non-magnetic and non-conducting so that applied field is not modified.

(ii) Magnetization in the ferrofluid is negligible compared to the applied magnetic field, i.e. $|\mathbf{M}| \ll|\mathbf{H}|$. 


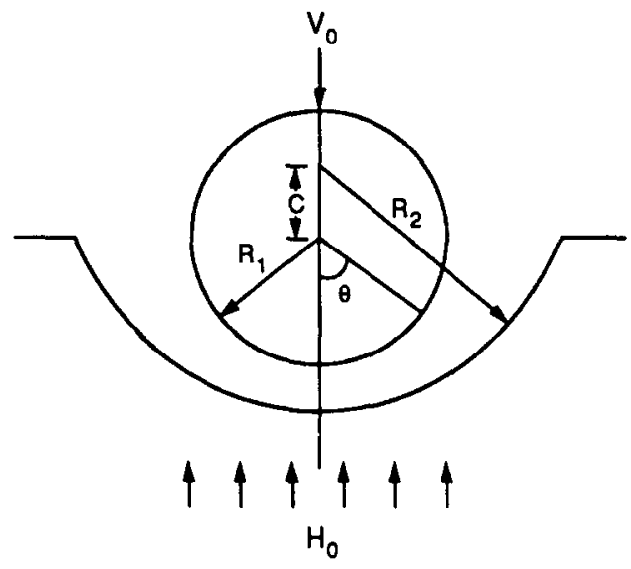

Fig. 1. Spherical bearing configuration.

(iii) The ferrofluid is saturated so that $M_{0}$ does not depend upon the applied magnetic field.

(iv) Thin film lubrication approximations are valid so that the derivatives of velocity, magnetization etc. along the film are negligible in comparison to their derivative across the film.

Thus, under the above assumptions, the equations governing steady viscous flow of ferrofluid $[11,12]$ reduce to the following form in the present casc;

$$
\begin{gathered}
-\frac{\mathrm{d} P}{R_{1} \mathrm{~d} \theta}+\eta \frac{\partial^{2} U}{\partial Z^{2}}+\frac{\mu_{0} H_{0}}{2} \frac{\partial M_{1}}{\partial Z}=0 \\
M_{1}=\frac{\tau_{B} M_{2}}{2} \frac{\partial U}{\partial Z}-\frac{\mu_{0} \tau_{B} \tau_{s}}{I} M_{1} M_{2} H_{0} \\
M_{2}=M_{0}-\frac{\tau_{B} M_{1}}{2} \frac{\partial U}{\partial Z}+\frac{\mu_{0} \tau_{B} \tau_{S}}{I} M_{1}^{2} H_{0}
\end{gathered}
$$

where $\mathbf{M}=\left(M_{1}, 0, M_{2}\right), U$ is the velocity in axial direction, $P$ is the pressure in the film, $H_{0}$ is applied magnetic field strength and is constant, $M_{0}$ is equilibrium magnetization, $\tau_{B}$ Brownian time relaxation parameter, $\tau_{S}$ is relaxation parameter due to rotation.

Boundary conditions for the velocity are

$$
U=0 \text { at } Z=0 \text { and } h
$$

and for pressure distribution are

$$
P=0 \quad \text { at } \quad \theta= \pm \frac{\pi}{2}
$$

Film thickness, $h$, is given by

$$
h=C(1-\varepsilon \cos \theta)
$$

where $\varepsilon(=e / C)$ is the eccentricity ratio and $e$ the eccentricity. Introducing the following non-dimensional variables

$$
p=\frac{P C^{3}}{\eta V_{0} R_{1}^{2}}, \quad u=\frac{U C}{V_{0} R_{1}}, \quad z=\frac{Z}{C}, \quad \bar{h}=\frac{h}{C}, \quad m_{1}=\frac{M_{1}}{M_{0}}, \quad m_{2}=\frac{M_{2}}{M_{0}}
$$

and non-dimensional parameters

$$
\tau=\frac{\tau_{B} V_{0} R_{1}}{C^{2}}, \quad N=\frac{\mu_{0} M_{0} H_{0} C^{2}}{\eta V_{0} R_{1}}, \quad A=\frac{\eta \tau_{S}}{I}
$$


the non-dimensional form of equations (10)-(14) is given by

$$
\begin{gathered}
-\frac{\mathrm{d} p}{\mathrm{~d} \theta}+\frac{\partial^{2} u}{\partial z^{2}}+\frac{N}{2} \frac{\partial^{2} m_{1}}{\partial z}=0 \\
m_{1}=\frac{\tau m_{2}}{2} \frac{\partial u}{\partial z}-A N \tau m_{1} m_{2} \\
m_{2}=1-\frac{\tau m_{1}}{2} \frac{\partial u}{\partial z}+A n \tau m_{1}^{2} \\
u=0 \quad \text { at } \quad z=0 \quad \text { and } \quad \tilde{h} \\
p=0 \quad \text { at } \quad \theta= \pm \pi / 2
\end{gathered}
$$

where

$$
\bar{h}=1-\varepsilon \cos \theta
$$

\section{Analysis}

Equations (17)-(19) are to be solved simultaneously to obtain velocity and pressure distribution. These equations are coupled non-linear equations and are not easily amenable to analytical solution. Therefore, we use perturbation technique to solve them. For representative values of $\tau_{B} \sim 10^{-6} \mathrm{~s}, \tau_{s} \sim 10^{-11} \mathrm{~s}, H_{0} \sim 10^{5} \mathrm{~A} / \mathrm{m}, M_{0} \sim 10^{4} \mathrm{~A} / \mathrm{m}, \mu_{0} \sim 10^{-7} \mathrm{~kg}-\mathrm{m} / \mathrm{s}^{2} \mathrm{~A}^{2}$, $I \sim 10^{-14} \mathrm{~kg} / \mathrm{m}[12]$ and $V_{0} \sim 10^{-3} \mathrm{~m} / \mathrm{s}, R_{1} \sim 10^{-1} \mathrm{~m}, C \sim 10^{-3} \mathrm{~m}$, we get $\tau \sim 10^{-4}, A \sim 1$, $N \sim 10^{4}$. So we use $\tau$ as a perturbation parameter. Thus, the following perturbation scheme is adopted;

$$
f=f_{0}+\tau f_{1}+\tau^{2} f_{2}+\tau^{3} f_{3}+0\left(\tau^{4}\right)
$$

where $f$ stands for various flow variables.

Using above perturbation scheme, and eliminating magnetization vector components of different orders, we obtain following equations governing velocity component $u_{i}$ of different orders:

$$
\begin{gathered}
-\frac{\mathrm{d} p_{0}}{\mathrm{~d} \theta}+\frac{\partial^{2} u_{0}}{\partial z^{2}}=0 \\
-\frac{\mathrm{d} p_{1}}{\mathrm{~d} \theta}+\frac{\partial^{2} u_{1}}{\partial z^{2}}+\frac{N}{4} \frac{\partial}{\partial z}\left[\frac{\partial u_{0}}{\partial z}\right]=0 \\
-\frac{\mathrm{d} p_{2}}{\mathrm{~d} \theta}+\frac{\partial^{2} u_{2}}{\partial z^{2}}+\frac{N}{4} \frac{\partial}{\partial z}\left[\frac{\partial u_{1}}{\partial z}-A N \frac{\partial u_{0}}{\partial z}\right]=0 \\
-\frac{\mathrm{d} p_{3}}{\mathrm{~d} \theta}+\frac{\partial^{2} u_{3}}{\partial z^{2}}+\frac{N}{4} \frac{\partial}{\partial z}\left[\frac{\partial u_{2}}{\partial z}-A N \frac{\partial u_{1}}{\partial z}+\left\{A^{2} N^{2}-\frac{1}{4}\left(\frac{\partial u_{0}}{\partial z}\right)^{2}\right\} \frac{\partial u_{0}}{\partial z}\right]=0
\end{gathered}
$$

Solving equations (24)-(27) along with the perturbed boundary conditions for $u$, i.e. $u_{i}=0$ at $z=0$ and $\bar{h}$, we get

$$
\begin{gathered}
u_{0}=\left(\frac{\mathrm{d} p_{0}}{\mathrm{~d} \theta}\right)\left(\frac{z^{2}-z \bar{h}}{2}\right) \\
u_{1}=\left[\frac{\mathrm{d} p_{1}}{\mathrm{~d} \theta}-\frac{N}{4} \frac{\mathrm{d} p_{0}}{\mathrm{~d} \theta}\right]\left(\frac{z^{2}-z \bar{h}}{2}\right) \\
u_{2}=\left[\frac{\mathrm{d} p_{2}}{\mathrm{~d} \theta}-\frac{N}{4}\left\{\frac{\mathrm{d} p_{1}}{\mathrm{~d} \theta}-N\left(\frac{1}{4}+A\right) \frac{\mathrm{d} p_{0}}{\mathrm{~d} \theta}\right\}\right]\left(\frac{z^{2}-z \bar{h}}{2}\right) \\
u_{3}=\left[\frac{\mathrm{d} p_{3}}{\mathrm{~d} \theta}-\frac{N}{4}\left\{\frac{\mathrm{d} p_{2}}{\mathrm{~d} \theta}-N\left(\frac{1}{4}+A\right) \frac{\mathrm{d} p_{1}}{\mathrm{~d} \theta}+N^{2}\left(\frac{1}{16}+\frac{A}{2}+A^{2}\right) \frac{\mathrm{d} p_{0}}{\mathrm{~d} \theta}\right\}\right]\left(\frac{z^{2}-z \bar{h}}{2}\right) \\
+\frac{N}{128}\left(\frac{\mathrm{d} p_{0}}{\mathrm{~d} \theta}\right)^{3}\left(2 z^{4}-4 z^{3} \bar{h}+3 z^{2} \bar{h}^{2}-z \bar{h}^{3}\right)
\end{gathered}
$$


Flow flux is given by

$$
Q=2 \pi R \sin \theta \int_{0}^{h} U \mathrm{~d} Z
$$

Also, it may be noted that the flow flux is the fluid displaced due to squeeze velocity between the two surfaces. Thus in the case of spherical bearing it is given by

$$
Q=\pi V_{0} R^{2} \sin \theta
$$

From equations (32) and (33), we get

$$
\int_{0}^{\bar{h}} u \mathrm{~d} z=\frac{\sin \theta}{2}
$$

Substitution of $u_{i}$ from equations (28)-(31) in perturbed equations as obtained from (34) yields the following differential equations for pressure distributions of different orders:

$$
\begin{gathered}
\frac{\mathrm{d} p_{0}}{\mathrm{~d} \theta}=-\frac{6 \sin \theta}{(1-\varepsilon \cos \theta)^{3}} \\
\frac{\mathrm{d} p_{1}}{\mathrm{~d} \theta}=-\frac{3 N}{2} \frac{\sin \theta}{(1-\varepsilon \cos \theta)^{3}} \\
\frac{\mathrm{d} p_{2}}{\mathrm{~d} \theta}=\frac{3 N^{2} A}{2} \frac{\sin \theta}{(1-\varepsilon \cos \theta)^{3}} \\
\frac{\mathrm{d} p_{3}}{\mathrm{~d} \theta}=-\frac{3 N^{3} A^{2}}{2} \frac{\sin \theta}{(1-\varepsilon \cos \theta)^{3}}+\frac{27 N}{40} \frac{\sin ^{3} \theta}{(1-\varepsilon \cos \theta)^{7}}
\end{gathered}
$$

Solving equations (35)-(38) with the perturbed boundary conditions for pressure, i.e. $p_{i}=0$ at $\theta= \pm \pi / 2$, we get pressure $p$ in the following form

$$
\begin{aligned}
p= & \frac{3}{\varepsilon}\left[1+\frac{N \tau}{4}\left\{1-N A+N^{2} A^{2} \tau^{2}\right\}\right]\left\{\frac{1}{(1-\varepsilon \cos \theta)^{2}}-1\right\} \\
& -\frac{27 N \tau^{3}}{40 \varepsilon}\left[\frac{1}{2}\left\{\frac{1}{2(1-\varepsilon \cos \theta)^{6}}-1\right\}-\frac{\cos 2 \theta}{4 \varepsilon(1-\varepsilon \cos \theta)^{6}}\right. \\
& \left.+\frac{\cos \theta}{5 \varepsilon(1-\varepsilon \cos \theta)^{5}}-\frac{1}{20 \varepsilon^{2}}\left\{\frac{1}{(1-\varepsilon \cos \theta)^{4}}-1\right\}\right]
\end{aligned}
$$

The dimensionless load capacity $\bar{W}$ is then given by

$$
\bar{W}=\frac{W C^{3}}{\eta V_{0} R_{1}^{4}}=\frac{6 \pi}{\varepsilon}\left[1+\frac{N \tau}{4}\left\{1-N A \tau+N^{2} A^{2} \tau^{3}\right\}\right] I_{1}-\frac{27}{20} \frac{N \pi \tau^{3}}{\varepsilon} I_{2}
$$

where

$$
\begin{gathered}
W=2 \pi R_{1}^{2} \int_{0}^{\pi / 2} P \sin \theta \cos \theta \mathrm{d} \theta \\
I_{1}=\int_{0}^{\pi / 2}\left\{\frac{1}{(1-\varepsilon \cos \theta)^{2}}-1\right\} \sin \theta \cos \theta \mathrm{d} \theta \\
I_{2}=\int_{0}^{\pi / 2}\left[\frac{1}{2}\left\{\frac{1}{2(1-\varepsilon \cos \theta)^{6}}-1\right\}-\frac{\cos 2 \theta}{4(1-\varepsilon \cos \theta)^{6}}\right. \\
\left.+\frac{\cos \theta}{5 \varepsilon(1-\varepsilon \cos \theta)^{5}}-\frac{1}{20 \varepsilon^{2}}\left\{\frac{1}{(1-\varepsilon \cos \theta)^{4}}-1\right\}\right] \sin \theta \cos \theta \mathrm{d} \theta
\end{gathered}
$$

In order to get squeeze time, we revert back to the dimensional form of equation (40), which is 
as follows:

$W=\frac{6 \eta \pi R^{4}}{\varepsilon C^{3}} V_{0}\left[1+\frac{\mu_{0} M_{0} H_{0} \tau_{B}}{4 \eta}\left\{1-\frac{\mu_{0} M_{0} H_{0} \tau_{B} \tau_{S}}{I}+\frac{\mu_{0}^{2} M_{0}^{2} H_{0}^{2} \tau_{B}^{2} \tau_{S}^{2}}{l^{2}}\right\}\right] I_{1}-\frac{27 \pi}{20} \frac{\mu_{0} M_{0} H_{0} \tau_{B}^{3} R_{1}^{6}}{C^{7}} I_{2} V_{0}^{3}$

On substituting $V_{0}=C(\mathrm{~d} \varepsilon / \mathrm{d} t)$ in equation (41) and non-dimensionalizing in the following manner:

$$
\bar{t}=t \frac{W C^{2}}{\eta R_{1}^{4}}, \quad N^{*}=\frac{\mu_{0} M_{0} H_{0} R_{1}^{4}}{W C^{2}}, \quad \tau^{*}=\tau_{B} \frac{W C^{2}}{\eta R_{1}^{4}}
$$

equation (41) can be rewritten in the following form:

$$
1=\frac{6 \pi}{\varepsilon}\left(\frac{\mathrm{d} \varepsilon}{\mathrm{d} \bar{t}}\right)\left[1+\frac{N^{*} \tau^{*}}{4}\left\{1-N^{*} A \tau^{*}+N^{* 2} A^{2} \tau^{* 2}\right\}\right] I_{1}-\frac{27 \pi}{20} \frac{N^{*} \tau^{* 3}}{\varepsilon}\left(\frac{R}{C}\right)^{2}\left(\frac{\mathrm{d} \varepsilon}{\mathrm{d} \bar{t}}\right)^{3} I_{2}
$$

To find squeeze time we first solve equation (43) for $\mathrm{d} \varepsilon / \mathrm{d} \bar{t}$, numerically, by giving initial approximation of $\mathrm{d} \varepsilon / d \bar{t}$ as $(\mathrm{d} \varepsilon / \mathrm{d} \bar{t}$ at $\tau=0)$ i.e. for non-ferromagnetic case. Then time $\bar{t}$ is obtained by using Simpson's rule:

$$
\bar{t}=\frac{\Delta \varepsilon}{3}\left(F_{0}+4 F_{1}+2 F_{2}+4 F_{3}+\cdots+2 F_{2 n-2}+4 F_{2 n-1}+F_{2 n}\right)
$$

where $F=1 /(\mathrm{d} \varepsilon / \mathrm{d} \bar{t})$ has been evaluated numerically at different mesh points ( $\Delta \varepsilon$ is the interval length).

\section{CONICAL BEARING}

In this section we shall study the flow of an incompressible ferrofluid in conical bearing for the case of pure squeezing (Fig. 2). A constant magnetic field is applied transversely to the motion of the fluid. The squeeze velocity $V_{0}$ is assumed constant in magnitude and direction.

Introducing the following non-dimensional variables:

$$
p=\frac{P h_{0}^{3}}{\eta V_{0} R^{2}}, \quad u=\frac{U h_{0}}{V_{0} R}, \quad x=\frac{X}{R}, \quad z=\frac{Z}{h_{0}}, \quad \bar{h}=\frac{h}{h_{0}},
$$

$R^{*}=R / h_{0}, m_{1}=M_{1} / M_{0}, m_{2}=M_{2} / M_{0} ; h_{0}$ is the initial film thickness; and non-dimensional parameters

$$
\tau=\frac{\tau_{B} V_{0} R}{h_{0}^{2}}, \quad N=\frac{\mu_{0} M_{0} H_{0} h_{0}^{2}}{\eta V_{0} R}, \quad A=\frac{\eta \tau_{S}}{I}
$$

the equations governing flow of ferrofluid under the assumptions (i)-(iv) are given in the



Fig. 2. Conical bearing configuration. 
non-dimensional form as follows:

$$
\begin{gathered}
-\frac{\mathrm{d} p}{\mathrm{~d} x}+\frac{\partial^{2} u}{\partial z^{2}}+\frac{N}{2} \frac{\partial m_{1}}{\partial z}=0 \\
m_{1}=\frac{\tau m_{2}}{2} \frac{\partial u}{\partial z}-N A \tau m_{1} m_{2} \\
m_{2}=1-\frac{\tau m_{1}}{2} \frac{\partial u}{\partial z}+N A \tau m_{1}^{2}
\end{gathered}
$$

The boundary conditions are:

$$
\begin{array}{lll}
u=0 & \text { at } & z=0 \\
p=0 & \text { at } & x=1 / \sin \alpha
\end{array}
$$

Analysis

Equations (45)-(47) are coupled non-linear equations which are solved using the perturbation technique, elaborated in Section 3. Following the analysis of Section 3, and solving the resultant equations, obtained on eliminating $m_{1}$ and $m_{2}$, with conditions $u_{i}=0$ at $z=0, \bar{h}$ we get

$$
\begin{gathered}
u_{0}=\left[\frac{\mathrm{d} p_{0}}{\mathrm{~d} x}\right]\left(\frac{z^{2}-z \bar{h}}{2}\right) \\
u_{1}=\left[\frac{\mathrm{d} p_{1}}{\mathrm{~d} x}-\frac{N}{4} \frac{\mathrm{d} p_{0}}{\mathrm{~d} x}\right]\left(\frac{z^{2}-z \bar{h}}{2}\right) \\
u_{2}=\left[\frac{\mathrm{d} p_{2}}{\mathrm{~d} x}-\frac{N}{4}\left\{\frac{\mathrm{d} p_{1}}{\mathrm{~d} x}-N\left(\frac{1}{4}+A\right) \frac{\mathrm{d} p_{0}}{\mathrm{~d} x}\right\}\right]\left(\frac{z^{2}-z \bar{h}}{2}\right) \\
u_{3}=\left[\frac{\mathrm{d} p_{3}}{\mathrm{~d} x}-\frac{N}{4}\left\{\frac{\mathrm{d} p_{2}}{\mathrm{~d} x}-N\left(\frac{1}{4}+A\right) \frac{\mathrm{d} p_{1}}{\mathrm{~d} x}+N^{2}\left(\frac{1}{16}+\frac{A}{2}+A^{2}\right) \frac{\mathrm{d} p_{0}}{\mathrm{~d} x}\right\}\right]\left(\frac{z^{2}-z \bar{h}}{2}\right) \\
+\frac{N}{128}\left(\frac{\mathrm{d} p_{0}}{\mathrm{~d} x}\right)^{3}\left(2 z^{4}-4 z^{3} \bar{h}+3 z^{2} \bar{h}^{2}-z \bar{h}^{3}\right)
\end{gathered}
$$

Flow flux is given by

$$
Q=2 \pi X \sin \alpha \int_{0}^{h} U \mathrm{~d} Z
$$

where $R$ is the radius of the base of the cone.

An alternative expression for flux is due to the displacement of the fluid between the squeezing surfaces. In the case of conical bearing this is given by

$$
Q=\frac{\pi X^{2} V_{0}}{\sin \alpha}
$$

where $\alpha$ is the semivertical angle of the cone.

From equations (53) and (54), we get

$$
\int_{0}^{\bar{h}} u_{0} \mathrm{~d} z=\frac{x}{2 \sin ^{2} \alpha}, \quad \int_{0}^{\bar{h}} u_{i} \mathrm{~d} z=0, \quad i=1,2,3
$$

Substituting equations (49)-(52) in equations (55), correspondingly, we get the differential equations for pressure of different orders, which on solving alongwith the boundary conditions for $p$ in perturbed form, gives

$$
p=\frac{3}{\bar{h}^{3} \sin ^{2} \alpha}\left[1+\frac{N \tau}{4}\left\{1-N A \tau+N^{2} A^{2} \tau^{2}\right\}\right]\left[\frac{1}{\sin ^{2} \alpha}-x^{2}\right]-\frac{81 N \tau^{3}}{160 \sin ^{6} \alpha} \bar{h}^{2}\left[\frac{1}{\sin ^{4} \alpha}-x^{4}\right]
$$


Load capacity in this case is given by

$$
W=2 \pi \sin ^{2} \alpha \int_{0}^{R / \sin \alpha} P X \mathrm{~d} X
$$

which on using equation (56) for pressure gives non-dimensional load as

$$
\bar{W}=\frac{W h_{0}^{3}}{\eta R^{4} V_{0}}=\frac{3 \pi}{2 \bar{h}^{3} \sin ^{4} \alpha}\left[1+\frac{N \tau}{4}\left\{1-N A \tau+N^{2} A^{2} \tau^{2}\right\}\right]-\frac{27}{80} \frac{\pi N \tau^{3} \bar{h}^{2}}{\sin ^{10} \alpha}
$$

Now to get squeeze time, we rewrite equation (57) in dimensional form; then, on substituting $V_{0}=-\mathrm{d} h / \mathrm{d} t$ in resultant equation and non-dimensionalizing in the following manner:

$$
\bar{t}=\frac{t W h_{0}^{2}}{\eta R^{4}}, \quad N^{*}=\frac{\mu_{0} M_{0} H_{0} R^{4}}{W h_{0}^{2}}, \quad \tau^{*}=\frac{\tau_{B} W h_{0}^{2}}{\eta R^{4}}
$$

we get

$$
-1=\frac{3 \pi}{2 \sin ^{4} \alpha} \frac{1}{\bar{h}^{3}}\left(\frac{\mathrm{d} \bar{h}}{\mathrm{~d} \bar{t}}\right)\left[1+\frac{N^{*} \tau^{*}}{4}\left\{1-N^{*} A \tau^{*}+N^{* 2} A^{2} \tau^{* 2}\right\}\right]-\frac{27}{80} \frac{\pi N^{*} R^{* 2} \tau^{* 3}}{\sin ^{10} \alpha} \breve{h}^{2}\left(\frac{\mathrm{d} \bar{h}}{\mathrm{~d} \bar{t}}\right)^{3}
$$

To find squeeze time, equation (59) is first solved for $\mathrm{d} \bar{h} / \mathrm{d} \bar{t}$ numerically by giving initial approximation of $\mathrm{d} \bar{h} / \mathrm{d} \bar{t}(\mathrm{~d} \bar{h} / \mathrm{d} \bar{t}$ at $\tau=0)$ for different values of $\bar{h}$. These values of $\mathrm{d} \bar{h} / \mathrm{d} \bar{t}$ are subsequently used to find squeeze time using Simpson's rule.

\section{RESULTS AND DISCUSSION}

The bearing characteristics obtained in Sections 3 and 4 contain the non-dimensional ferrofluid parameters $N, \tau, A$ [as defined in equations (16) and (44)] and $N^{*}, \tau^{*}$ [defined in equations (42) and (58)]. Taking representative values of various quantities, we obtain $N, N^{*} \sim 10^{4} ; \tau, \tau^{*} \sim 10^{-4}$ and $A \sim 1$ in both the cases.

It may be noted that for a given bearing configuration any change in applied magnetic field strength $H_{0}$ is reflected through $N$ or $N^{*}$. Similarly a change in $\tau\left(\tau^{*}\right)$ is a consequence of change in Brownian relaxation time parameter $\tau_{B}$, whereas increase in $A$ can be either due to increase in $\tau_{S}$ (relaxation time of particle rotation due to frictional resistance of fluid) or decrease in $I$ (sum of moment of inertia per unit volume). Obviously, therefore as $\tau$ and $N$ increase, departure from Newtonian fluid theory will be enhanced. This effect is elaborated in the following results.

In the absence of applied magnetic field the parameter $N$ becomes zero and the results similar to Newtonian theory are obtained. Another limiting case is that when Brownian relaxation time parameter $\tau_{B}$ is zero, which again gives rise to Newtonian case, however when $A=0$, results do not reduce to the Newtonian case. The effects of various dimensionless parameters on spherical and conical bearing characteristics have been numerically studied. As the effect of ferrofluid parameters in the two cases is observed to be qualitatively similar, we present here results for spherical bearings only.

\section{(i) Pressure distribution and load capacity}

Non-dimensional pressure distribution $p$ is plotted versus $\theta$ in Figs 3 and 4 for different values of $N, A$ and $\tau$. Since the pressure curve is symmetric about $\theta=0$ in spherical bearings so its variation has been shown from $\theta=0$ to $\pi / 2$. In Fig. 3, variation of pressure distribution for $N$ and $\tau$ is shown for $A=0$ and $\varepsilon$ (eccentricity ratio) $=0.3$, whereas in Fig. 4 variation of $A$ and film thickness is shown for fixed values of $N\left(=0.5 \times 10^{4}\right)$ and $\tau\left(=0.5 \times 10^{-4}\right)$. It is seen from Fig. 3 that the qualitative behaviour of the pressure distribution remains similar to that in the Newtonian case (corresponding to $N=0$ ) for various values of $N$ and $\tau$. However, there is a 


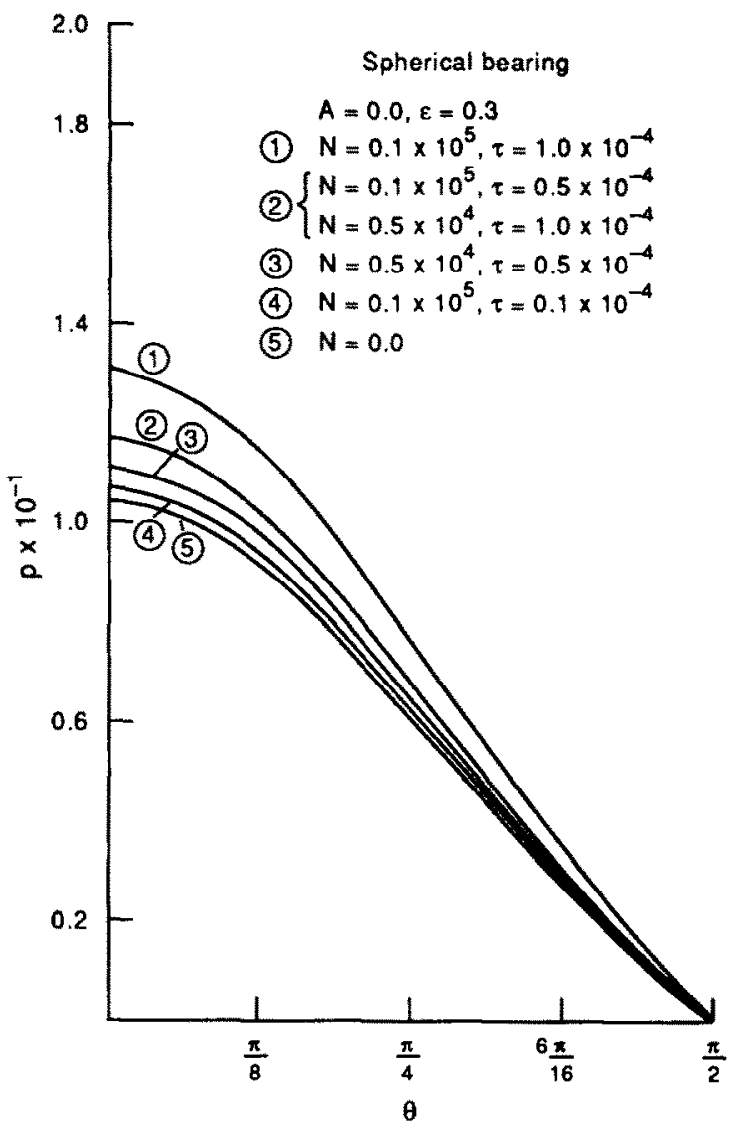

Fig. 3. Variation of non-dimensional pressure $(p)$ vs $\theta$.

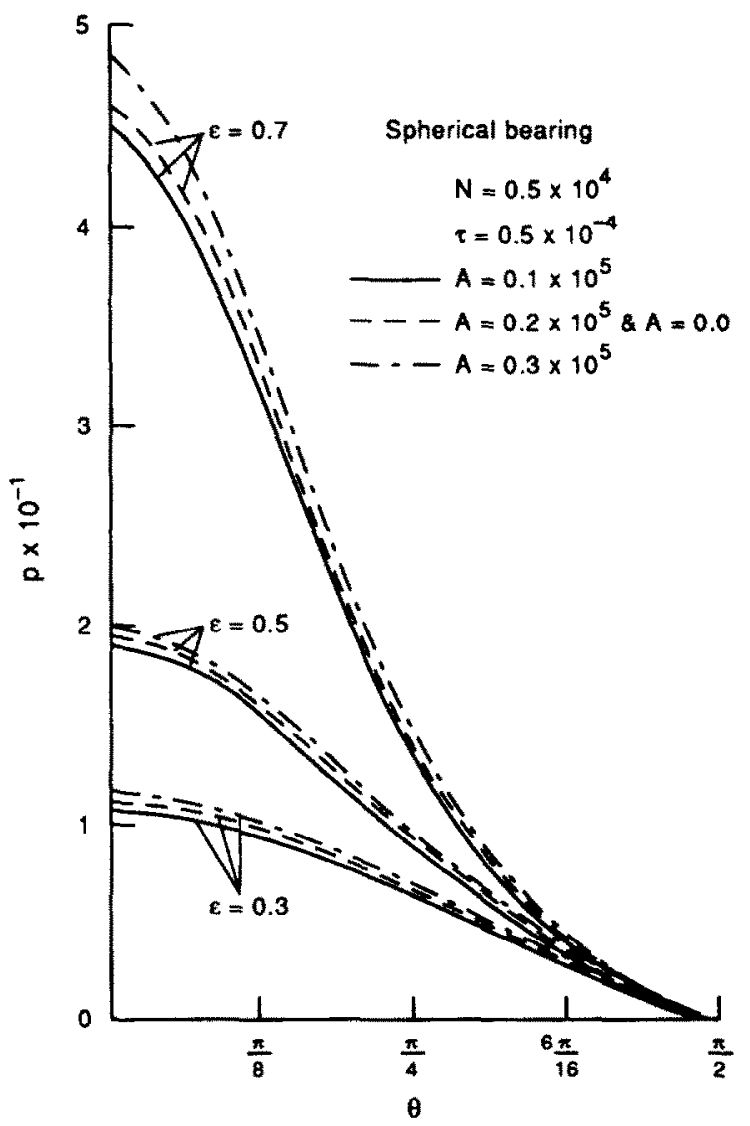

Fig. 4. Variation of nondimensional pressure $(p)$ vs $\theta$. 


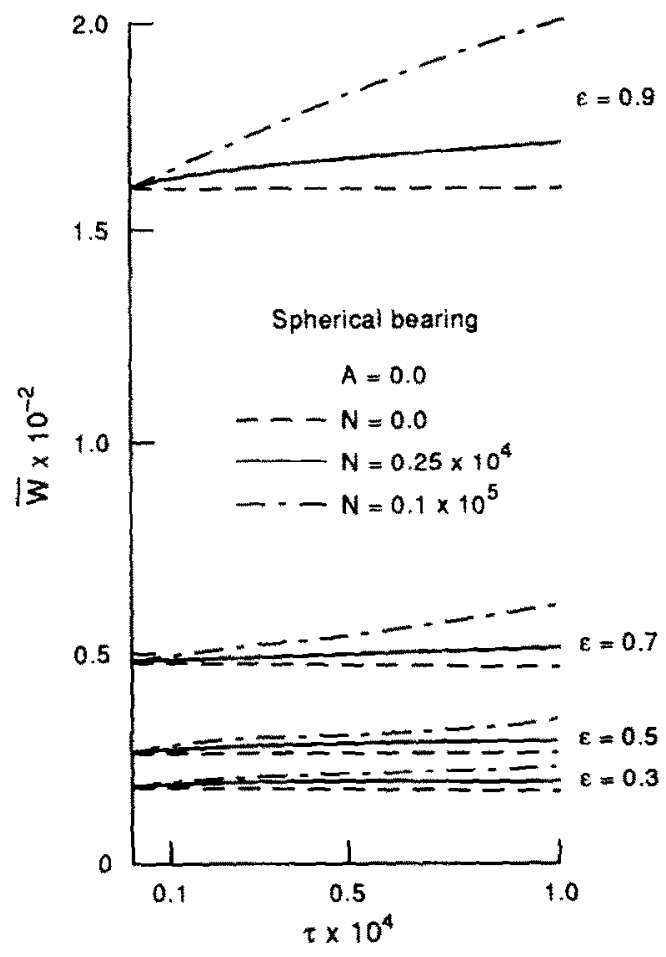

Fig. 5. Variation of non-dimensional load $(\bar{W})$ vs $t$.

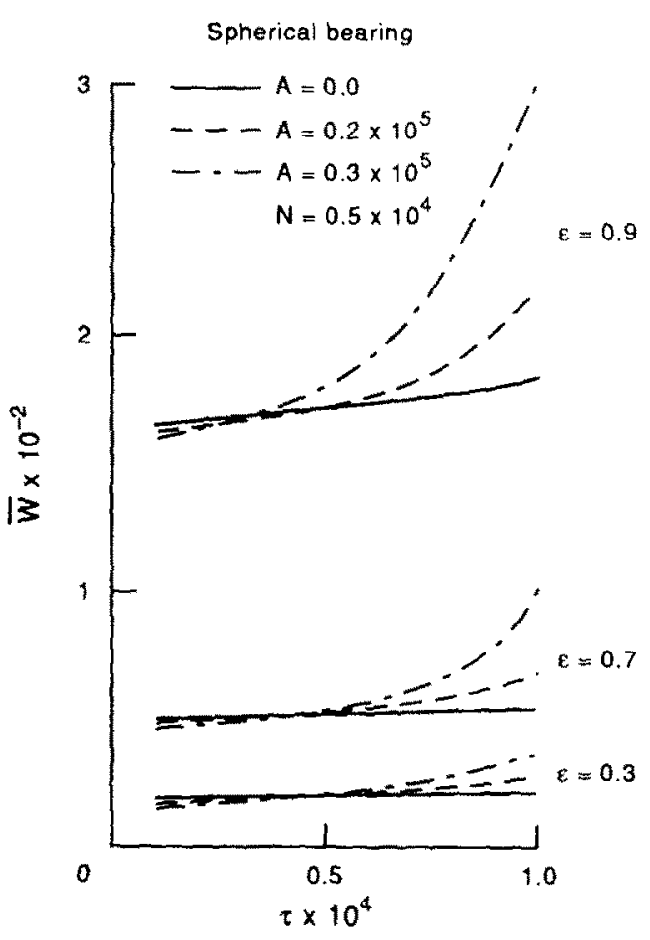

Fig. 6. Variation of non-dimensional load capacity $(\bar{W})$ vs $\tau$.

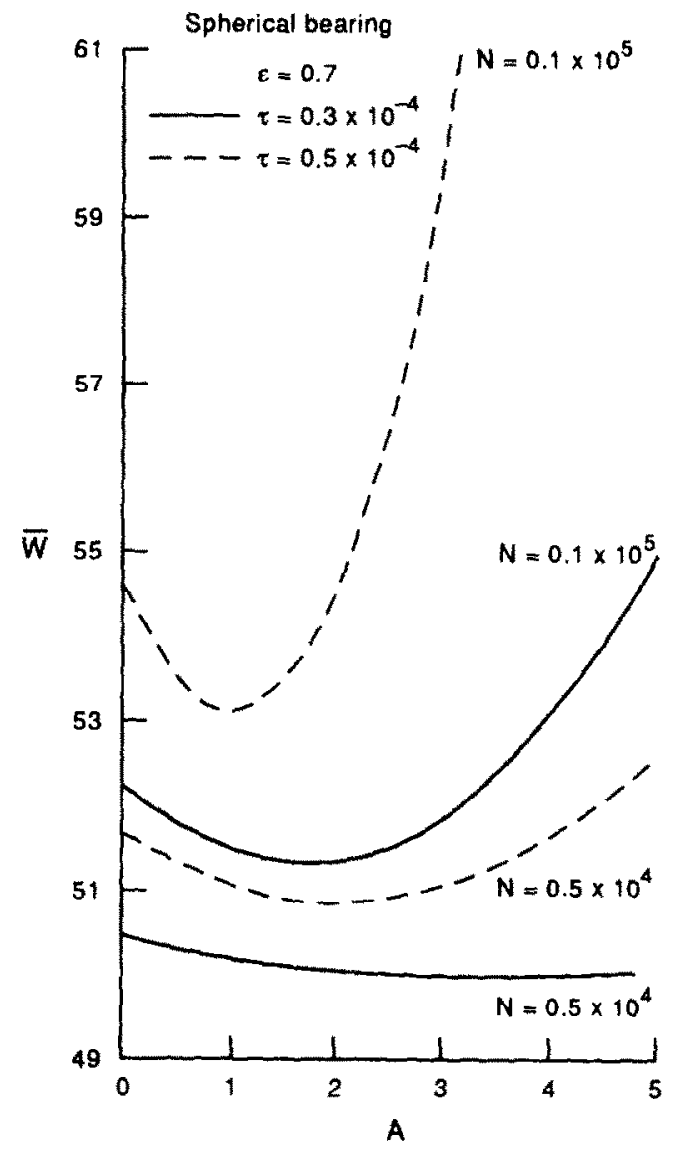

Fig. 7. Variation of non-dimensional load $(W)$ vs $A$. 
significant quantitative increase, especially in the pressure peak region, as $N$ and $\tau$ increase. It is observed on comparing curves (1), (2) and (2), (3) of Fig. 3 that the effect of increase of $\tau$ gets enhanced as the applied magnetic field becomes stronger.

From Fig. 4 it is seen that as film thickness decreases pressure distribution increases. It is also noted from Fig. 4 that as $A$ increases pressure initially decreases and then shows an increasing trend. This effect will be further highlighted in Fig. 7 for load.

Non-dimensional load capacity is plotted vs $\tau$ in Figs 5 and 6 and vs $A$ in Fig. 7. In Fig. 5 effect of $N$ on load capacity is shown for $A=0$, while in Fig. 6 effect of $A$ on load capacity is shown for fixed $N$. It is seen from Fig. 5, that the increase in load capacity with respect to $\tau$ remains almost linear. Further, as film thickness decreases (i.e. $\varepsilon$ increases) increase in load capacity is more with $N$ and $\tau$. From Fig. 6, it is noted that for fixed $N$ and film thickness, as $A$ increases load capacity decreases initially, then increases slowly up to approximately $\tau=$ $0.3 \times 10^{-4}$ there after increase in load capacity is sharp with respect to $\tau$. The variation of load vs $\tau$ does not remain linear for higher values of $A$.

The effect of variation of $A$ on load capacity is further elaborated in Fig. 7. For fixed $N$ and $\tau$, as $A$ increases load capacity decreases initially, attains a minimum for some $A$ and increases subsequently. This effect is obvious from the expressions of load capacity which gives that minimum value of $\bar{W}$ is at

$$
A=\frac{1}{2 N \tau}
$$

This increase or decrease in load capacity with respect to $A$ becomes steeper as $N$ and $\tau$ increase.

\section{(ii) Squeeze time}

Time height relationship is shown in Fig. 8 for different values of $N^{*}$ and $\tau^{*}$. It may be noted here that squeeze time increases with the increase in $N^{*}$ and $\tau^{*}$, which is a consequence of increase in pressure and load capacity with the increase in $H_{0}$ and $\tau_{B}$. Further it is observed that for higher values of $N^{*}$ and $\tau^{*}$, time-height relationship has a predominant departure from

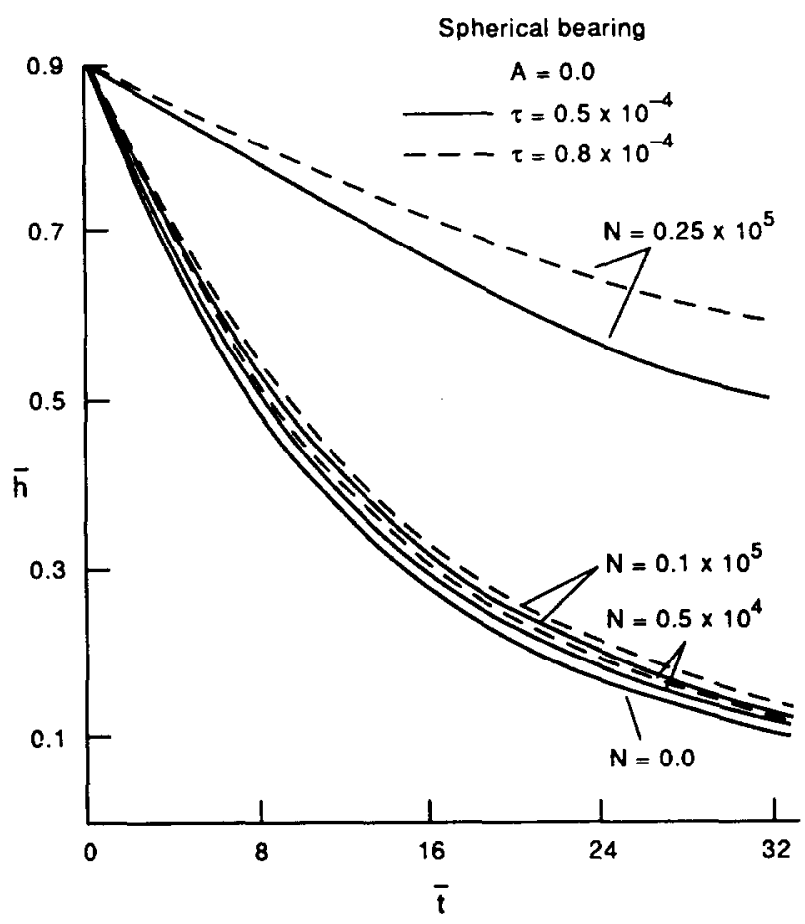

Fig. 8. Time-height relationship. 


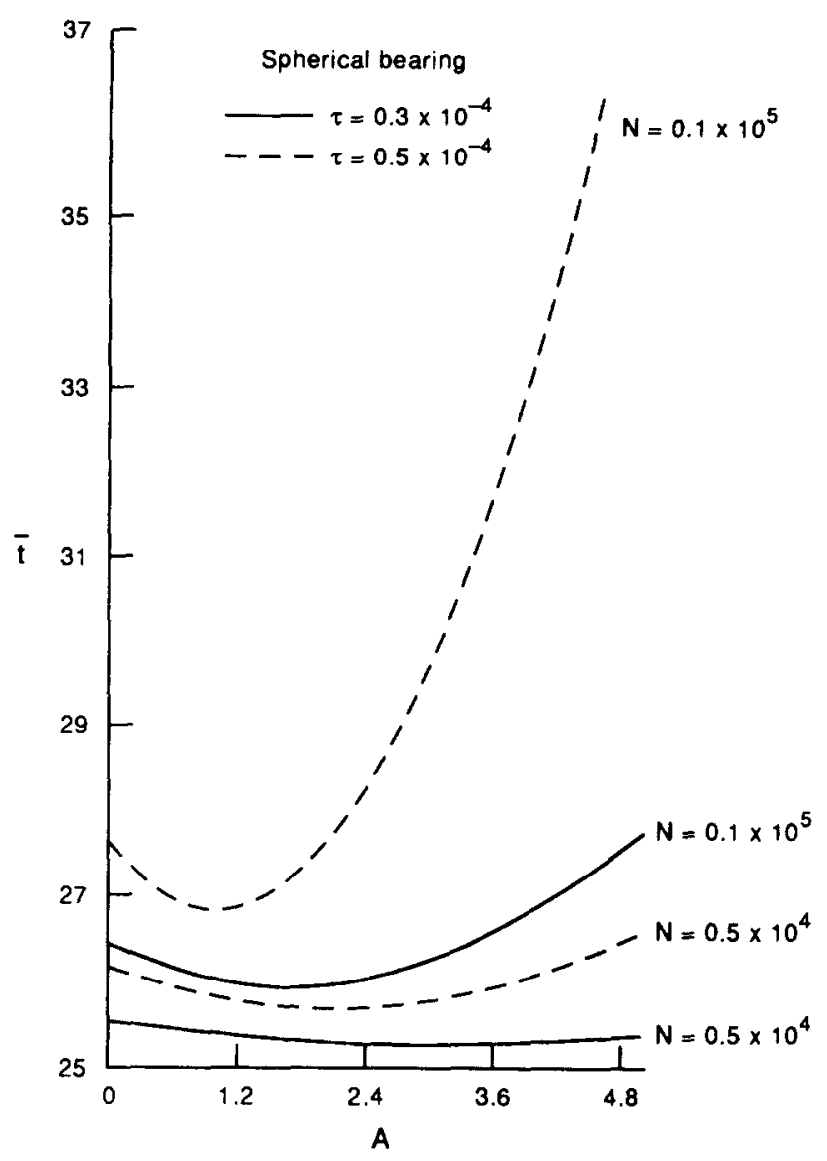

Fig. 9. Squeeze times $(\bar{t})$ vs $A$ as $\varepsilon$ changes from 0.5 to 0.9 .

the Newtonian case. Squeeze time vs $A$ is shown in Fig. 9, for different values of $N^{*}$ and $\tau^{*}$ as the upper plate approaches the lower one from $\bar{h}=0.9(\varepsilon=0.5)$ to $\bar{h}=0.1 \quad(\varepsilon=0.9)$. The behaviour of squeeze time with $A$ remains similar to that in the case of load. By increasing $N^{*}$ and $\tau^{*}$, the decrease and increase in squeeze time becomes sharper as $A$ increases.

\section{REFERENCES}

[1] E. N. MOZGOVOI, E. YA BLUM and A. O. TSEBERS, Magnetohydrodynamics 9, 52 (1973).

[2] I. E. TARAPOV, Magnitnaya Gidrodinamika 8, 19 (1972).

[3] J. S. WALKER and J. D. BUCKMASTER, Int. J. Engng Sci. 17, 1171 (1982).

[4] N. TIPEI, J. Lub. Tech. (ASME) 104, 510 (1982).

[5] P. D. S. VERMA, Int. J. Engng Sci. 24, 395 (1986).

[6] V. K. AGARWAL, Wear 107, 133 (1986).

[7] F. SORGE, J. Tribology (ASME) 109, 77 (1987).

[8] J. B. SHUKLA and D. KUMAR, JMMM 65, 375 (1987).

[9] D. KUMAR and P. CHANDRA, in Proc. of Cont. Mech. and its Applications (Edited by G. A. C. GRAHAM and S. K. MALIK), p. 741. Hemisphere, New York (1989).

[10] J. L. NEURINGER and R. E. ROSENSWEIG, Phys. Fluids 7, 1926 (1964).

[11] M. I. SHLIOMIS, Sov. Phys. JETP 34, 1291 (1972).

[12] S. KAMIYAMA, K. KOIKE, N. IIZUKA, Bull. ISMF. 22, 1205 (1979). 